\title{
The Effect of Economic Deprivation on Adolescents' Happiness: The Mediating Effects of Family Conflict and Adolescents' Depression and Anxiety
}

\author{
Sae-Young Han, Ah-Reum Han \\ Department of Child Development \& Intervention, Ewha Womans University, Seoul, Korea
}

$$
\begin{aligned}
& \text { 경제적 박탈이 청소년의 행복감에 미치는 영향: 가족갈등과 청소년의 우울/ } \\
& \text { 불안의 매개효과 }
\end{aligned}
$$

한세영, 한아름

이화여자대학교 아동학과

\begin{abstract}
Objective: The main purpose of this study was to examine the effects of economic deprivation on adolescents' happiness and the mediating effects of family conflict on adolescents' depression and anxiety.

Methods: Data from the 7th wave of Korea Welfare Panel Study (KWPS) and child supplementary study were used. A total of 512 adolescents and their households were included. Data were analyzed by path analysis using AMOS 21.0.

Results: First, economic deprivation were positively related to family conflict, and family conflict was positively related to adolescents' depression and anxiety, whereas economic deprivation, family conflict and adolescents' depression/anxiety were negatively associated with adolescents' happiness. Second, economic deprivation had a direct effect on adolescents' happiness. Third, family conflict played a mediating role in the relationship between economic deprivation and adolescents' happiness. Finally, both family conflict and adolescents' depression/anxiety played a mediating role in the relationship between economic deprivation and adolescents' happiness.

Conclusion: Results of this study indicate that economic deprivation, family conflict, and adolescents' depression/anxiety are important factors lowering adolescents' happiness. These findings also suggest that for promoting adolescents' happiness, it is necessary to provide not only support policies focusing on economic deprivation but also intervention programs to strengthen relationships within family members and prevent adolescents' depression/anxiety, creating a happier life for them.
\end{abstract}

Keywords: economic deprivation, family conflict, depression/anxiety, happiness, adolescent$$
\text { 서론 }
$$

우리나라의 청소년들의 삶이 행복과 거리가 있음을 많은 연구

Corresponding Author: Ah-Reum Han, Department of Child Development \& Intervention, Ewha Womans University, 52, Ewhayeodate-gil, Seodaemun-gu, Seoul 03760, Korea

E-mail: arumi0701@naver.com
}

들이 밝히고 있다. OECD국가의 아동.청소년의 행복지수를 비교한 연구(Youm \& Kim, 2017)에 따르면, 우리나라의 청소년 들의 주관적인 행복지수는 $\mathrm{OECD}$ 국가 중 최하위권을 유지하

(C)The Korean Association of Child Studies

This is an Open Access article distributed under the terms of the Creative Commons Attribution Non-Commercial License (http:// creativecommons.org/licenses/by-nc/4.0) which permits unrestricted noncommercial use, distribution, and reproduction in any medium, provided the original work is properly cited. 
는 것으로 나타났다. 청소년들은 학업에 대한 부담감, 미래에 대한 불안함으로 인해 낮은 행복감을 느낀다고 하였으며(K.J. Kim \& Kim, 2014), 연령이 증가하면서 청소년들의 행복감은 점차 감소되어, 고등학교시기에 가장 낮은 행복감을 느끼는 것 으로 보고되었다(Ahn, 2012; Lim, Baek, Kim, \& Kang, 2012).

행복감은 주관적으로 느끼는 긍정적인 정서경험으로, 주관 적 안녕감(subjective well-being)으로 표현되기도 한다(Diener, 2000). 청소년의 행복감에 관한 연구들을 살펴보면, 청소년 들의 행복감이 높을수록 긍정적인 사회적 관계를 형성하였 으며, 학업에 대한 만족감과 자아존중감, 자아탄력성, 낙관적 태도가 높은 것으로 나타났다(Ahn, 2012; Cheng \& Furnham, 2003; Kang, 2015; Y. H. Kim, 2010; E. Park \& Baek, 2017; Raboteg-Saric \& Sakic, 2014; Shin, Jeon, \& Yoo, 2010; UusitaloMalmivaara \& Lehto, 2013). 이러한 연구들은 청소년 시기의 행복감은 긍정적 발달의 중요한 지표가 된다는 점에서 청소년 시기의 행복한 삶은 매우 중요하다고 주장하였다. 따라서 청 소년들의 행복한 삶을 위해, 어떠한 요인이 청소년들의 행복 한 삶을 저해하고 있는지 규명하기 위해서 개인적 요인, 환경 적 요인 등 관련된 다양한 요인들을 살펴볼 필요가 있다.

특히 가정의 경제적 어려움은 아동과 청소년의 신체적, 정서적, 사회적 발달에 상당한 영향을 미쳐(Brooks-Gunn \& Duncan, 1997), 보다 낮은 행복감을 경험할 수 있다. 경제적 어 려움은 자녀에게 직접적으로 스트레스를 유발하는 환경을 제 공하기도 하지만, 부부 간 또는 부모-자녀간의 갈등을 유발하 고 이러한 가족관계에서의 갈등이 자녀의 심리적 부적응과 낮 은 행복감으로 이어질 수 있다. 본 연구에서는 경제적 요인이 청소년 행복감에 영향을 미치는 경로를 살펴보고자 하였으며, 그 과정에서 사회 맥락적 요인으로 가족구성원 간 갈등, 개인 심리적 요인으로 우울 및 불안이 어떠한 영향을 미치는가를 확인함으로써, 청소년의 행복에 미치는 변인들 간의 구조적 관계를 규명하고자 하였다.

가정의 경제적 어려움과 자녀의 발달과의 관계를 살펴본 연구는 주로 가계소득에 초점을 두고 경제적 어려움이 자녀 의 발달에 미치는 영향을 살펴보았다(Brown, 2004; Hanson \& Chen, 2007; Letourneau, Duffett-Leger, Watson, \& Young-Morris, 2013). 경제수준이 높은 가정의 청소년들은 경제수준이 낮은 가정의 청소년들에 비해 주관적 행복감이 높은 것으로 나타 났으며(Yoo \& Lee, 2013), 경제적 수준이 낮고, 주거 빈곤기간 이 길수록 청소년들은 낮은 행복감을 느끼는 것으로 나타났 다(Gariepy, Elgar, Sentenac, \& Barrington-Leigh, 2017; H. Lee, 2016; Lim \& Kim, 2016; S.-Y. Park \& Lee, 2013).
그러나 몇몇 연구자들은 경제적으로 어려움에 처한 가정의 현실을 제대로 이해하고, 적절한 정책적 방안을 모색하기 위 해서는 기존의 가계 소득만으로 경제적 어려움을 설명하는데 한계가 있음을 주장하였다. 이는 경제적 어려움을 경험하는 가정의 자녀들은 교육, 주거, 의료 등의 다양한 영역에서의 박 탈을 경험함에도 불구하고, 가계소득은 이를 반영하지 못하기 때문이다. 따라서 소득 이외의 교육, 의료, 주거, 결식 또는 영 양불균형 등 생활에서 경험하는 다양한 영역의 경제적 박탈경 험을 고려할 필요가 있음을 주장하였다(E. Kim, 2015; Joung, Choi, Lee, \& Ha, 2013). 경제적 박탈과 청소년의 행복감, 주관 적 안녕감과의 관계를 다룬 선행연구를 살펴보면, 가정 내 재 정적 어려움으로 인해 필수품 구입의 어려움, 여가생활의 제 한 등 개인적 욕구가 좌절되고 경제적 압박감을 경험하는 경 제적 박탈은 청소년의 웰빙에 장기적으로 부정적 영향을 미치 는 것으로 나타났다(Walper, 2009)

이와 달리 가정의 경제적 변인이 자녀의 행복감에 미치는 영향이 크지 않다는 연구결과도 있다. 이러한 연구들에서는 가정의 경제적 변인은 자녀의 행복감을 설명하는 주된 변수가 아님을 밝히고 있다. 즉, 가구의 소득수준 또는 물질적 결핍에 비해 자녀의 개인 심리적 요인과 가족관계, 또래관계와 같은 사회관계적 요인이 자녀의 행복감에 미치는 영향이 큰 것으로 나타났으며(Holder \& Coleman, 2008; Holder \& Klassen, 2010; McAuley \& Layte, 2012; Suh \& Jung, 2014), 소득수준이나 경제 적 박탈과 같은 경제적 변인이 자녀의 행복감에 직접적인 영 향을 미치기 보다는 개인 심리적 요인 또는 부모, 또래 등의 사 회관계적 요인을 통해 미치는 간접적인 영향이 더 큰 것으로 나타났다(Sung \& Kim, 2013). 이러한 결과들은 경제적 박탈경 험이 어떠한 매개경로를 통해 청소년의 행복감에 영향을 미치 는지 파악해야 할 필요성을 시사한다.

경제적 박탈로 인해 영향을 받을 수 있는 사회 맥락적 요인 에는 가족관계가 있으며, 가족관계는 청소년의 행복감을 예측 하는 주요 변수로도 볼 수 있다. 먼저, 경제적 어려움으로 인해 구성원들은 높은 스트레스를 경험하게 되고, 이는 가족 구성 원 간의 부정적인 상호작용과 갈등을 유발할 가능성이 높다. 선행연구에서 이를 지지하고 있는데, 청소년 자녀가 지각하 는 가계 빈곤수준이 높을수록 가족갈등수준이 높은 것으로 나 타났으며(P. Noh \& Yoon, 2007), 경제적으로 곤란하여 발생하 는 의식주에서의 결핍과 개인의 욕구가 좌절되는 경험은 가족 갈등 수준을 높이는 것으로 나타났다(Wadsworth \& Compas, 2002). 또한, 경제적 어려움으로 인해 식생활과 자녀교육, 주 거, 의료와 건강에서의 문제를 경험하고 경제적으로 압박감을 
경험하는 것은 가족구성원들 간의 갈등과 적대감을 높이는 것 으로 나타났다(J.-I. Kim, 2009).

한편, 청소년이 지각한 행복과 행복의 조건을 살펴본 U. Kim, Park 과 Baak (2012)의 연구에 따르면, 청소년들은 가정이 화목할 때 가장 행복하다고 느끼는 반면, 가족 간의 의견충돌, 부모자녀 갈등 등의 가정에 불화가 있을 때 가장 불행하다고 느끼는 것으로 나타났다. 이러한 결과는 청소년들의 행복감을 결정하는 핵심요소로 가족구성원간의 화목한 관계라는 점을 시사한다. 또한, 긍정적인 가족구성원들 간의 관계는 청소년 의 안녕감을 높이는(H.-J. Kim, 2017) 반면, 가족구성원 간의 적대감과 긴장과 갈등은 자녀의 안녕감에 부정적 영향을 미치 는 것으로 나타났다(J.-M. Lee \& Lee, 2007; Joronen \& ÅstedtKurki, 2005).

뿐만 아니라 가족 구성원들 간의 갈등은 청소년의 우울과 불안에 큰 영향을 미친다. H.-M. Yoon (2005)의 연구에서는 가 족갈등으로 인한 스트레스가 아동과 청소년의 우울 및 불안을 높였으며, 가족 구성원들 간의 갈등과 역기능적인 상호작용, 구성원들간의 낮은 유대감은 청소년들의 우울 및 불안에 부정 적인 영향을 미치는 것으로 나타났다(Buist et al., 2017; Chung \& Choi, 2004; Y.-H. Kim \& An, 2008; P. Noh \& Yoon, 2007). 뿐 만 아니라 가족 간의 갈등은 1 년 이후의 청소년의 우울에도 유의한 영향을 미치는 것으로 나타났다(Sheeber, Hops, Alpert, Davis \& Andrew, 1997).

이러한 우울과 불안은 많은 연구들에서 행복감 높은 관 련성을 보이는 것으로 알려져 왔다(Jung \& Cho, 2009; S. S. Kim, 2016; Uusitalo-Malmivaara \& Lehto, 2013; Wilkinson, \& Walfold, 1998; Neuman, van Lier, Friins, Meeus, \& Koot, 2011; Chaplin, 2006). 우울 및 불안과 행복감의 인과관계를 밝힌 연 구가 많지는 않으나 행복감과 안녕감에 대한 우울과 불안의 영향력을 살펴본 연구들에서는 우울과 불안이 개인의 행복감 과 안녕감을 예측하는 주요 변수임을 일관되게 밝히고 있다 (Derikan-Eiron et al, 2011; S.-Y. Park \& Lee, 2013; Shim, 2014; Sihn et al, 2010; Zehng, 2016; G. J. Yoon, 2012). 결과적으로 청 소년의 우울과 불안은 주관적 행복감에 유의한 영향을 미칠 것으로 짐작해볼 수 있으나, 청소년을 대상으로 두 변인과의 관계를 살펴본 연구가 드물기에 본 연구에서 두 변인간의 관 계를 검증하고자 하였다.

한편, 청소년기 우울 및 불안과 행복감은 성에 따라 다르 다고 보고되는데, 여자 청소년이 남자청소년에 비해 우울 및 불안을 높게 경험하는 반면(Kang, 2013; Shin, Chung, \& Kim, 2012), 행복감의 경우에는 남자 청소년이 여자청소년에 비
해 높은 것으로 나타났다(S.-Y. Park \& Lee, 2013; Suh \& Jung, 2014). 뿐만 아니라 학업성적도 청소년기의 우울 및 불안과 행복감에 유의한 영향을 미치는 변수로 보고되고 있다(C.-R. Noh \& Kim, 2012; S.-Y. Park \& Lee, 2013). 이러한 결과들은 청 소년기 우울 및 불안과 행복감과 같은 심리적 요인에 성과 학 업성적을 고려해야함을 시사하며, 이에 본 연구에서는 성과 학업성적을 통제하여 살펴보고자 하였다.

종합해보면, 경제적 박탈경험은 청소년의 행복감에 직접적 인 영향을 미칠 수 있는 환경이 될 수 있을 뿐 아니라, 가족구 성원간의 갈등수준과 청소년의 우울 및 불안을 통해 청소년의 행복감에 이를 것으로 예측해 볼 수 있다. 경제적 어려움으로 인해 경제적 압박과 스트레스 느끼고 우울, 불안, 좌절 등의 부 정적 정서를 경험함으로써 부부 간 또는 부모-자녀간의 역기 능적 상호작용과 갈등이 촉진되고(Conger \& Elder, 1994), 이 러한 가족구성원들 간의 갈등이 청소년 자녀의 심리적 적응에 부정적 영향을 미치고 있음을 밝히고 있다(Conger, Ge, Elder, Lorenz, \& Simons, 1994). 따라서 본 연구에서는 이러한 관계를 검증함으로써 청소년의 건강한 성장과 행복한 삶, 그리고 그 가족의 안녕을 돕기 위한 정책적, 실천적 방안을 마련하는데 필요한 기초자료를 제공하고자 한다. 이를 위해 본 연구에서 설정한 연구문제는 다음과 같다.

\section{연구 문제 1}

경제적 박탈은 청소년의 행복감에 직접적인 영향을 미치는가?

\section{연구 문제 2}

경제적 박탈은 가족갈등과 청소년의 우울 및 불안을 통해 행복 감에 간접적인 영향을 미치는가?

\section{연구방법}

\section{연구대상}

본 연구는 한국복지패널(Korean Welfare Panel Study [KWPS]) 7 차년도 가구용 자료와 아동부가조사 자료를 사용하였다. 한국 복지패널은 2006년에 1차년도 조사를 시작하였으며, 아동 부 가조사는 3 년을 주기로 $1,4,7,10$ 차 년도에 실시되었다. 아동 부가조사에서는 1차년도에 패널조사 가구원 중 초등학교 4-6 학년 아동 대상으로 처음 실시하였다. 7차년도(2012년)에서는 1 차년도 응답자(2006년 당시 초등학교 4-6학년 아동)와 4차년 
도 응답자(2009년 당시 중학교 1-3학년 아동)가 조사에 참여 하였으며, 7차년도 조사 당시 연령은 만 16-18세로, 중퇴, 미진 학 등의 이유로 학교를 다니지 않는 청소년들도 조사에 포함 되었다(Korea Institute for Health and Social Affeair [KIHASA] \& Seoul National University Institute of Social Welfare, 2013). 본 연 구에서는 가구용 조사, 아동부가 조사 데이터를 결합(merge) 하여 분석하였다. 고등학생 연령을 연구대상으로 선정한 이유 는 많은 선행연구에서 자녀의 연령이 증가할수록 행복감이 낮 아지며, 초등학생와 중학생에 비해 고등학생의 행복감이 가장 낮은 것으로 밝히고 있어, 고등학생의 행복감에 영향을 미치 는 요인을 파악해 볼 필요성이 있기 때문이었다. 분석에 포함 된 청소년은 512명으로, 남자 청소년은 264명(51.6\%), 여자 청 소년은 248명(48.4\%)이며, 이들의 학년을 살펴보면 고1 173명 (33.8\%), 고2 165명(32.2\%), 고3 158명(30.9), 현재 휴학, 중퇴, 또는 미진학 16 명 $(3.1 \%)$ 이다. 가구의 특성을 살펴보면 가구의 특성을 살펴보면, 저소득 가구는 120 가구(23.4\%), 일반가구는 392 가구(76.6\%)로 나타났다. 이는 한국복지패널에서는 가구 균등화된 경상소득을 기준으로 $60 \%$ 미만인 경우 저소득가구, 그렇지 않은 가구는 일반가구로 분류하였다.

\section{연구도구}

\section{경제적 박탈}

경제적 박탈을 측정하기 위해 한국복지패널 가구용 자료에 서‘돈이 없거나 부족하여 겪을 수 있는 상태에 대한 경험 여부’ 를 묻는 13 문항을 사용하였다. 박탈의 영역은 결식(경제적 어 려움 때문에 먹을 것을 살 돈이 없어서 배가 고픈데도 먹지 못 한 경험), 집세 미납(2달 이상 집세가 밀리거나 낼 수 없어 집 을 옮긴 경험), 난방 제한(돈이 없어 겨울에 난방을 못한 경험), 교육비 미납(돈이 없어서 자녀의 공교육비를 한 달 이상 주지 못한 경험), 의료서비스 제한(돈이 없어서 본인이나 가족이 병 원에 갈 수 없었던 경험), 신용불량경험(가구원 중 신용불량자 가 된 경험자 여부) 등의 영역을 묻는 문항으로 구성되어 있으 며, 본 연구에서는 박탈경험이 있는 경우(1점)와 박탈경험이 없는 경우(0점)로 하고, 문항의 값을 전체 합산하였다. 점수가 높을수록 경제적 박탈경험이 많은 것을 의미한다.

\section{가족갈등}

가족갈등은 한국복지패널 가구용 자료에서 가족구성원들 간
의 갈등과 관련된 5 문항을 사용하였다. 문항의 예로 "우리 가 정에서는 의견 충돌이 잦다.", "가족원들이 가끔 너무 화가 나 서 물건 등을 집어 던진다.” 등이 있으며, 전혀 그렇지 않다(1 점)에서 매우 그렇다(5점)로 응답한다. 일부 문항을 제외하고 역코딩하여, 점수가 높을수록 가족 내 갈등수준이 높음을 의 미한다. 신뢰도 계수(Cronbach's $\alpha$ )는 .76으로 나타났다.

\section{우울 및 불안}

우울 및 불안을 측정하기 위해 한국복지패널 아동부가조사에 서는 Achenbahc (1991)가 개발한 아동청소년 평가척도(Child Behavior Checklist)를 Oh, Lee, Hong과 Ha (1998)가 번안한 척 도 $\mathrm{K}-\mathrm{CBCL}$ 중 우울 및 불안 14 문항을 사용하였다. 문항의 예 로 "아무도 나를 사랑하지 않는다고 불평하거나 생각한다.", “지나치게 겁이 많거나 불안해한다.” 등이 있으며, 각 문항에 대해 전혀 아니다(1점)에서 자주 그렇다(3점)으로 응답한다. 각 문항을 역코딩하여, 점수가 높을수록 우울 및 불안정도가 높음을 의미한다. 신뢰도 계수(Cronbach's $\alpha$ )는 .87 이다.

\section{행복감}

행복감을 측정하기 위하여 한국복지패널 아동부가조사에 서는 Institute for Social Development Studies, Yonsei University (2010)의 척도를 사용하였다. 문항의 예로 "나는 건강하다고 생각한다.”, “나는 삶에 만족한다.” 등의 문장을 포함하며, 각 문항에 대해 전혀 그렇지 않다(1점)에서 매우 그렇다(5점)로 응답한다. 일부 문항을 제외하고 문항을 역코딩하여, 점수가 높을수록 행복감이 높음을 의미한다. 신뢰도 계수(Cronbach's $\alpha)$ 는 .68이다.

\section{통제변수: 성과 학업 성적}

본 연구에서는 청소년기 우울 및 불안과 행복감과 같은 정서 적 발달이 성에 따라 다르게 나타나며(Kang, 2013; S.-Y. Park \& Lee, 2013; Shin et al, 2012), 학업성적에 영향을 받는다는 선행 연구(C.-R. Noh \& Kim, 2012; S.-Y. Park \& Lee, 2013)들을 바탕 으로 성과 학업성적을 통제변수에 포함하였다. 성별의 경우, 남자청소년(1점), 여자청소년(0점)으로 더미변수화하여 분석 하였다. 학업성적의 경우, 전과목 평균에 대해, 아주못함(1점) 에서 아주 잘함(5점)으로 응답한 문항을 사용하였으며, 점수 가 높을수록 학업성적이 높음을 의미한다. 


\section{자료분석}

본 연구에서 자료를 분석하기 위해 SPSS 20.0프로그램(IBM Co., Armonk, NY)을 사용하여 빈도분석 및 기술통계와 측정도 구의 신뢰도분석을 실시하였다. 또한, 연구변인들 간의 관계를 파악하기 위해 청소년의 성과 학업성적을 통제하여 상관분석 을 실시하였다. 경제적 박탈경험이 가족갈등과 우울 및 불안을 통해 청소년의 행복감에 영향을 미치는 경로를 검증하기 위해 AMOS 21.0 프로그램(IBM Co., Armonk, NY)을 사용하여 경로 분석을 실시하였다. 마지막으로 간접효과의 유의성을 확인하 기 위하여 부트스트래핑(bootstrapping)을 사용하였다.

\section{연구결과}

본 연구의 주요변인인 경제적 박탈경험, 가족갈등, 청소년의 우울 및 불안, 행복감의 기술통계 분석 및 변인들 간의 상관분 석을 실시한 결과는 Table 1 과 같다. 먼저, 변인들의 정규성 가 정이 충족되는지를 확인하기 위해 왜도 및 첨도를 살펴본 결 과, 경제적 박탈경험은 왜도 6.27 , 첨도 56.14로 왜도 절대값 3 미만, 첨도 절대값 10 미만이라는 정규성 기준(Kline, 2015)을 충족하지 않고 분포가 치우친 형태로 나타났다. 따라서 정규 성 가정을 충족하기 위해 경제적 박탈경험 변인을 제곱근 변 환하여 분석하였다.

청소년의 성과 학업 성적을 통제한 후 편상관계수를 산출 한 결과, 경제적 박탈경험은 가족갈등과 유의한 정적상관 $(r=$ $.20, p<.001)$ 을, 청소년의 행복감과 유의한 부적상관 $(r=-.11$, $p<.05)$ 을 보여, 경제적 박탈경험을 많이 경험할수록 가족 구 성원 간 갈등수준이 높고, 청소년의 행복감이 감소하는 것으 로 나타났다. 가족갈등은 청소년의 우울 및 불안과 유의한 정 적 상관 $(r=.15, p<.01)$ 을, 청소년의 행복감과는 유의한 부적 상관 $(r=-.17, p<.001)$ 을 보여, 가족갈등수준이 높을수록 청소
Table 1

Descriptive Statistic Analysis and Correlations Among the Research Variables

\begin{tabular}{lcccc}
\hline Variables & 1 & 2 & 3 & 4 \\
\hline 1. Economic deprivation & - & & & \\
2. Family conflict & $.20^{* * *}$ & - & & \\
3. Depression/Anxiety & .02 & $.15^{* *}$ & - & \\
4. Happiness & $-.11^{*}$ & $-.17^{* * *}$ & $-.44^{* * *}$ & - \\
\hline$M$ & .23 & 1.77 & 1.33 & 3.70 \\
$S D$ & .52 & .53 & .33 & .73 \\
Skewness & 2.43 & .96 & 1.40 & -.18 \\
Kurtosis & 6.36 & 1.67 & 1.75 & -.35 \\
\hline
\end{tabular}

Note. Economic deprivation is presented as square root value. ${ }^{*} p<.05 .{ }^{* *} p<.01 .{ }^{* * *} p<.001$.

Table 2

Model Fit Indices of Research Model

\begin{tabular}{cccccc}
\hline$\chi^{2}$ & $d f$ & $p$ & NFI & CFI & RMSEA \\
\hline 2.718 & 3 & .437 & .989 & 1.000 & .000 \\
\hline
\end{tabular}

년의 우울 및 불안은 증가하고, 행복감은 감소하는 것으로 나 타났다. 청소년의 우울 및 불안은 행복감과 유의한 부적상관 $(r=-.44, p<.001)$ 을 보여, 청소년들의 우울 및 불안이 증가할 수록, 행복감이 감소하는 것으로 나타났다.

다음으로 경제적 박탈경험이 가족갈등과 청소년의 우울 및 불안을 매개로 하여 청소년의 행복감에 영향을 미치는 경 로를 검증하기 위해 청소년의 성과 학업성적을 통제하여 경로 분석을 실시하였다. Table 2에서 보듯이, 연구모형의 적합도를 살펴본 결과, $\mathrm{NFI}=.989, \mathrm{CFI}=1.000, \mathrm{RMSEA}=.000$ 으로 적 절한 모형의 기준을 충족하였다.

각 변인 간의 경로계수는 Table 3 과 Figure 1 과 같다. 먼저, 통제변수인 청소년의 성과 학업성적이 우울 및 불안(각각 $\beta$ $=-.23, \beta=-.12, p<.01)$ 과 행복감 $($ 각각 $\beta=.09, \beta=.17, p<.05$ ) 에 유의한 영향을 미치는 것으로 나타났다. 다음으로 청소년

Table 3

Path Coefficients of Research Model

\begin{tabular}{llccc}
\hline \multicolumn{1}{c}{ Paths } & $B$ & $\beta$ & $S E$ & C.R. \\
\hline Economic deprivation $\rightarrow$ Family conflict & .21 & .21 & .04 & $4.77^{* * *}$ \\
Economic deprivation $\rightarrow$ Happiness & -.11 & -.08 & .05 & $-1.96^{*}$ \\
\hline Family conflict $\rightarrow$ Depression/Anxiety & .09 & .14 & .03 & $3.383^{* * *}$ \\
Family conflict $\rightarrow$ Happiness & -.12 & -.09 & .05 & $-2.309^{*}$ \\
\hline Depression/Anxiety $\rightarrow$ Happiness & -.92 & -.42 & .09 & $-10.691^{* * *}$ \\
\hline
\end{tabular}

${ }^{*} p<.05 .{ }^{* * *} p<.001$. 


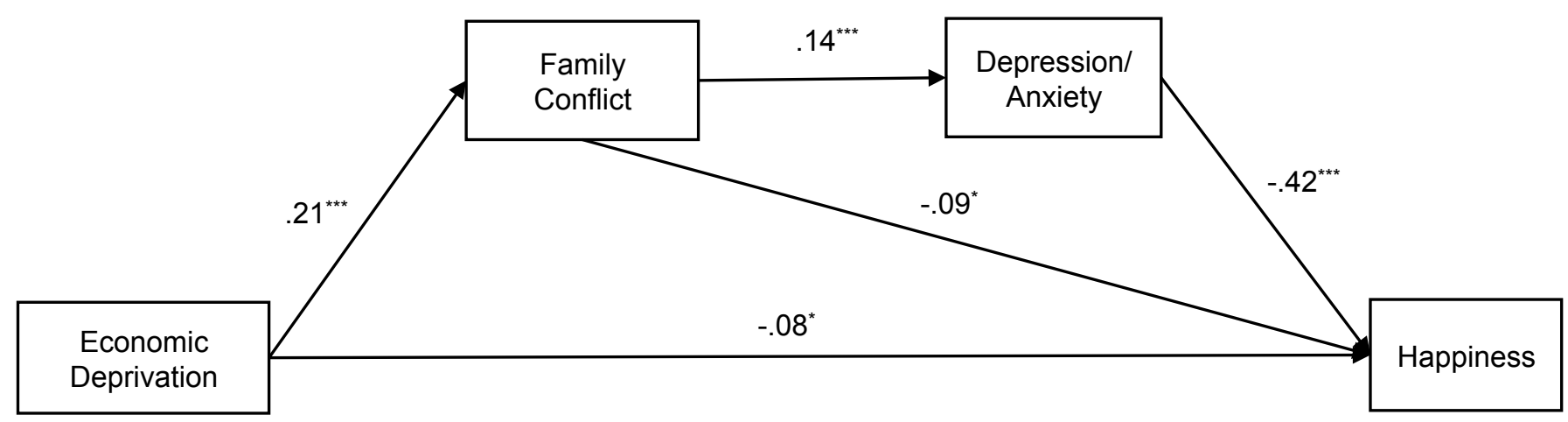

Figure 1. Pathways from economic deprivation to adolescents' happiness through family conflict and adolescents' depression/anxiety. ${ }^{*} p<.05 .{ }^{* * *} p<.001$.

Table 4

Direct, Indirect and Total Effects of Research Model

\begin{tabular}{|c|c|c|c|c|c|}
\hline \multicolumn{3}{|c|}{ Paths } & Direct & Indirect & Total \\
\hline Economic deprivation & $\rightarrow$ & Family conflict & $.21^{* * *}$ & - & $.21^{* * *}$ \\
\hline Economic deprivation & $\rightarrow$ & Depression/Anxiety & - & $.03^{* *}$ & $.03^{* *}$ \\
\hline Family conflict & & & $.14^{* * *}$ & - & $.14^{* * *}$ \\
\hline Economic deprivation & $\rightarrow$ & Happiness & $-.08^{*}$ & $-.03^{* *}$ & $-.11^{* *}$ \\
\hline Family conflict & & & $-.09^{*}$ & $-.06^{* *}$ & $-.15^{* *}$ \\
\hline Depression/Anxiety & & & $-.42^{* * *}$ & - & $-.42^{* * *}$ \\
\hline
\end{tabular}

${ }^{*} p<.05{ }^{* *} p<.01 .{ }^{* * *} p<.001$.

의 성과 학업성적을 통제한 상태에서, 경제적 박탈경험이 가 족갈등 $(\beta=.21, p<.001)$ 과 청소년의 행복감 $(\beta=-.08, p<.05)$ 에 이르는 경로, 가족갈등이 청소년의 우울 및 불안 $(\beta=.14$, $p<.001)$ 과 청소년의 행복감 $(\beta=-.09, p<.05)$ 에 이르는 경로, 청소년의 우울 및 불안이 행복감에 이르는 경로 $(\beta=-.42, p<$ .001)가 모두 유의한 것으로 나타났다.

이를 구체적으로 연구변인들 간의 직, 간접효과 및 총효과 를 살펴본 결과는 Table 4에 제시하였다. 경제적 박탈경험은 가족갈등에 직접적인 영향을 미치며 $(\beta=.21, p<.001)$, 청소 년의 행복감에는 직접적인 영향 $(\beta=-.08, p<.05)$ 뿐만 아니라 가족갈등과 청소년의 우울 및 불안을 통해 간접적인 영향 $(\beta=$ $-.03, p<.01)$ 을 미치는 것으로 나타났다. 가족갈등은 청소년 의 우울 및 불안에 직접적인 영향을 미치고 $(\beta=.14, p<.001)$, 청소년의 행복감에 직접적인 영향 $(\beta=-.09, p<.05)$ 뿐만 아니 라 청소년의 우울 및 불안을 통해 간접적인 영향 $(\beta=-.06, p<$ .01)을 미치는 것으로 나타났다. 마지막으로 청소년의 우울 및 불안은 행복감에 직접적인 영향을 미치는 것으로 나타났다 $(\beta$ $=-.42, p<.001)$. 즉, 경제적 박탈경험이 청소년의 행복감 감소 에 직접적인 영향을 미칠 뿐만 아니라, 경제적 박탈경험은 가
족구성원간의 갈등을 유발하고, 이는 청소년의 우울과 불안을 높이며, 결과적으로 행복감 감소에 영향을 미치는 것으로 나 타났다.

\section{논의 및 결론}

본 연구에서는 한국복지패널을 이용하여 청소년의 행복감에 영향을 미칠 수 있는 개인, 가족, 경제적 요인들 간의 관계를 살펴보고자, 성과 학업성적을 통제한 상태에서 경제적 박탈경 험이 가족갈등과 청소년의 우울 및 불안을 통해 행복감에 영 향을 미치는 경로를 검증하였다. 본 연구의 주요 연구결과 및 논의는 다음과 같다.

첫째, 경제적 박탈경험이 청소년의 행복감에 직접적으로 영향을 미치는 것으로 나타났다. 즉, 경제적으로 박탈된 경험 으로 인해 청소년들의 행복감이 감소하는 것으로 나타났다.

이는 경제적 박탈경험이 많을수록 심리적 부적응을 경험하고, 안녕감이 감소한다는 선행연구(J. Kim, You, \& Song, 2015; S. Park, 2013)와 가정의 경제적 어려움이 청소년의 행복과 안녕 
감과에 유의한 영향을 미친다는 선행연구(Gariepy et al., 2017; H. Lee, 2016; S.-Y. Park \& Lee, 2013)와 일치하는 결과이다. 열 악한 주거환경에서의 생활, 교육기회의 제한, 의료기관 이용의 제한 등 기본생활양식에서의 박탈경험은 청소년의 좌절감, 심 리적 압박감, 안전한 생활에 부정적 영향을 미칠 수 있어 전반 적인 삶의 질이 저하될 수 있다. 뿐만 아니라 욕구좌절의 경험 으로 청소년들은 또래아이들과 비교하여 상대적 박탈감, 좌절 감, 낮은 자아존중감 등의 심리적 고통을 경험할 수 있고 결과 적으로 전반적인 삶의 행복감이 낮아진 것으로 해석해 볼 수 있다. 이러한 결과는 청소년의 행복한 삶을 위한 하나의 방안 으로 경제적 박탈을 완화하기 위한 대책이 필요함을 시사한다.

둘째, 경제적 박탈경험은 가족갈등을 통해 청소년의 행복 감에 간접적으로 영향을 미치는 것으로 나타났다. 즉, 경제적 박탈경험은 가족 구성원 간 갈등을 유발하고, 이러한 가족 내 갈등이 청소년의 행복감을 감소시키는 것으로 나타났다. 이러 한 결과는 경제적 어려움과 빈곤수준이 가족갈등과 높은 관 련성을 보이며(P. Noh \& Yoon, 2007), 경제적 어려움이 가족 구성원 간의 갈등에 직접적인 영향을 미친다는 연구결과(J.-

I.. Kim, 2009; Wadsworth \& Compas, 2002)를 지지하며, 가족 구성원 간의 갈등과 불화가 청소년의 불행과 낮은 심리적 안 녕감과 관련이 있다는 연구결과(Joronen \& Åstedt-Kurki, 2005; U. Kim, Park, \& Baak, 2012; J.-M. Lee \& Lee, 2007)와 일치하 는 결과이다. 경제적 어려움으로 인해 기본적인 생활욕구(식 사, 난방, 의복, 의료 등)에서의 박탈을 경험을 경험한 가정에 서는 경제적 문제로 부부간의 갈등이 증가할 뿐 아니라, 부모 가 우울, 심리적인 압박감, 적대감 등의 심리적 부적응을 경험 함으로써 자녀에게 온정적으로 대하는데 어려움이 있을 수 있 다. 이와 같은 가정 내 박탈경험과 함께 가족갈등을 지속적으 로 경험하는 청소년들은 가족 내에서 충분한 안정감을 얻지 못하고, 자신의 삶이 불우하고 불행하다고 느꼈을 것으로 해 석해볼 수 있다. 이러한 결과는 경제적 박탈로 인해 개인의 욕 구와 좌절되고 심리적으로 어려운 상황 속에서도 가족 구성원 들 사이에서 발생하는 갈등을 보다 원만하게 해결하고, 서로 정서적으로 지지하고 애정적인 관계를 맺도록 돕는 것이 필요 하며, 이러한 화목한 가족관계를 위한 노력으로 인해 청소년 들의 행복감도 높아질 수 있음을 시사한다.

셋째, 경제적 박탈경험은 가족갈등과 청소년의 우울 및 불 안을 매개로 하여 청소년의 행복감에 간접적인 영향을 미치는 것으로 나타났다. 즉, 경제적 박탈경험은 가족 구성원간의 갈 등을 증가시키며, 높은 가족 갈등수준은 청소년의 우울과 불 안수준을 높이며, 결과적으로 행복감의 감소로 이어지는 것
으로 나타났다. 이러한 결과는 경제적 어려움을 경험할수록 가족갈등이 증가하고, 이러한 가족 구성원 간의 갈등과 역기 능적인 상호작용이 청소년의 우울 및 불안수준이 증가한다 는 선행연구(Buist et al., 2017; Chung \& Choi, 2004; Y.-H. Kim \& An, 2008; P. Noh \& Yoon, 2007; Sheeber et al., 1997; H.-M. Yoon, 2005)와 이러한 높은 우울 및 불안수준이 낮은 행복감으 로 이어진다는 선행연구(Chaplin, 2006; Derikman-Eiron et al., 2011; Jung \& Cho, 2009; S.S. Kim, 2016; Neuman et al., 2011; S.-Y. Park \& Lee, 2013; Sihn et al., 2010; Uusitalo-Malmivaara \& Lehto, 2013; Wilkinson \& Walfold, 1998)를 지지하는 결과로 볼 수 있다. 낮은 소득과 물리적 자원의 결핍으로 인해 가족 구 성원들 간의 갈등이 증가할 수 있는데, 이러한 구성원들 간의 갈등을 경험하는 청소년 자녀들은 우울과 불안을 느낄 수 있 다. 부부 간의 갈등은 이를 목격하는 것만으로도 자녀의 우울 과 불안을 유발 할 수 있다. 뿐만 아니라 부부간의 갈등으로 인 해 자녀에게 지지이고 온정적인 양육을 제공할 수 있는 심리 적 자원이 제한될 수 있다, 그 결과 자녀에게 부정적인 양육을 제공하고 자녀와의 역기능적인 상호작용이 이루어질 수 있으 며, 그 결과 자녀는 우울과 불안을 느낄 수 있을 것으로 유추해 볼 수 있다. 또한 부모 자신의 심리적 자원의 제한으로 인해 자 녀에게 충분한 정서적 지지지를 제공하는데 어려움이 있어, 자녀의 우울과 불안은 보다 증가하며. 이러한 높은 수준의 우 울과 불안을 경험하는 전반적인 삶에서 낮은 행복감을 경험한 것으로 해석해볼 수 있다. 따라서 청소년들의 행복한 삶을 위 해서는 가족 구성원간의 갈등이 자녀의 우울과 불안수준을 높 이고, 나아가 전반적인 삶의 행복감을 저해할 수 있음을 인지 하여, 화목한 가족관계를 맺도록 돕는 노력과 함께 청소년의 우울과 불안을 다루고 해소하기 위한 개입이 필요하다.

본 연구에서 주목할 점은 경제적 박탈경험이 청소년의 행 복감에 직접적으로 미치는 영향보다 상대적으로 가족 내 갈 등과 우울 및 불안수준이 더 큰 영향을 미치고 있다는 점이다. 이러한 결과는 경제적으로 박탈된 경험 그 자체보다는 이로 인해 야기되는 가족 구성원들 간의 갈등, 그리고 청소년 자녀 의 우울과 불안수준이 청소년의 행복감에 보다 중요한 요인 으로 작용하고 있음을 시사하는 것이라 볼 수 있다. 이는 경제 적 지위, 소득 등의 가정의 경제수준보다는 자녀의 기질 또는 사회성 등의 개인내적 요인과 부모의 양육, 학교환경 및 유대 감 등의 사회관계적 요인이 행복감에 더 큰 영향을 미친다는 선행연구들(Holder \& Coleman, 2008; Holder \& Klassen, 2010; McAuley \& Layte, 2012; Suh \& Jung, 2014)들과 가정 경제적 환 경이 청소년의 행복감에 직접적으로 영향을 미치기 보다는 개 
인의 심리적 특성 또는 사회관계적 요인을 통해 간접적으로 영향을 미친다는 선행연구(Sung \& Kim, 2013)와 유사한 결과 라 볼 수 있다. 이와 같이 경제적 박탈이 행복감에 미치는 직접 적인 영향보다 가족갈등과 청소년의 우울과 불안을 통해 행복 감에 영향을 미치는 영향이 크다는 본 연구의 결과는 경제적 박탈을 경험하는 청소년을 도울 때, 경제적 불안정함으로 인 해 유발된 가족 내 갈등 양상을 파악하고, 이로 인해 청소년이 겪는 심리적 요인에 보다 초점을 둔 개입 방안을 모색할 필요 가 있음을 시사한다.

이와 같은 결과를 토대로 본 연구는 청소년의 행복한 삶을 위해서는 경제적 박탈을 줄일 수 있도록 돕는 국가차원의 정 책적 지원이 필요할 뿐만 아니라, 가족 구성원간의 갈등을 원 만하게 해결하도록 도움으로써 청소년의 심리적 적응을 돕는 방안을 모색할 필요가 있다는 시사점을 제공하였다. 먼저, 경 제적 어려움에서 비롯된 다차원적 생활영역에서의 박탈을 해 소하기 위하여, 최저생계비 강화 등의 소득지원 차원을 넘어, 낙후된 주거환경 개선, 의료 보험 확대, 결식 가정지원, 자녀교 육 지원등의 다각적 차원의 사회보장 서비스 제도가 마련되고 강화되어야 할 것이다.

또한, 경제적 박탈경험으로 인한 가족갈등은 가족기능의 약화 뿐만 아니라 가족의 해체, 자녀의 행동문제 등의 사회문 제로 이어질 수 있으므로 예방적 차원에서 가족기능을 강화하 고 가족갈등을 원만히 해결하고 유대감을 높일 수 있는 가족 단위의 상담 서비스와 가족 캠프 등의 지역사회 프로그램을 개발하고 제공될 필요가 있다. 이를 위해 지역 내 건강가정지 원센터나 사회복지관과 민간의 상담기관과의 연계가 필요하 며, 서비스가 제공된 이후에도 지속적인 사후관리가 이루어져 야 할 필요가 있다. 특히 경제적 박탈을 경험하는 가족의 경우, 한부모 가족, 조손가족, 다문화 가족 등 다양한 유형의 가족이 포함되는 경우가 많아 가족 유형과 실질적 욕구에 적절한 프 로그램이 개발되고 제공될 필요가 있다. 청소년들의 우울과 불안 등의 정신건강을 돕기 위해서는 학교 뿐 아니라 학교와 지역사회가 연계하여 상담과 집단 프로그램이 실시될 필요가 있다.

본 연구에서는 가정의 경제적 환경을 단순히 소득수준이 아닌, 낮은 소득의 결과로 나타날 수 있는 다양한 삶의 영역에 서의 결핍을 의미하는 '박탈'개념으로 접근하였다. 이는 경제 적으로 어려운 가정은 식생활, 주고, 의료, 교육 등 생활 전반 에서 결핍을 경험하고, 기본적인 욕구와 필요가 좌절되고 있 음을 반영하고 있으므로, 보다 포괄적인 경제적 상황을 반영 하고 있기 때문이다. 따라서 최저생계보장비와 같은 경제적
지원뿐 만이 아니라, 영양, 의료보장, 주거개선, 교육지원 등을 함께 지속적으로 지원할 수 있는 정책의 필요성을 제시하는 근거를 제공했다는데 연구의 의의가 있다.

본 연구에서의 한계점과 후속연구를 위한 제언은 다음과 같다. 먼저, 경제적 박탈로 영양, 의료, 교육 등의 내용 등 다차 원적인 영역을 포함하고 있으나, 본 연구에서는 단순 합산하 여 분석함으로써 각 영역의 상대적인 영향까지는 살펴보지 못 했다. 보다 구체적인 정책적 함의를 도출하기 위해서 추후 연 구에서는 박탈경험의 각 영역을 세분화하여 영향력을 살펴 보는 것이 필요하다. 또한 본 연구에서 사용한 패널데이터 자 료에서는 우울과 불안을 하나의 변인으로 측정하고 있어 두 심리적 변인을 구분하여 살펴보는데 한계가 있었다. 그러나 우울과 불안을 큰 범주에서 유사한 속성을 가진 변인으로 보 는 선행연구도 많으나(Alexon \& Birmaher, 2001; Cummings, Caporino, \& Kendall, 2014; King, Ollendick, \& Gullone, 1991), 한편으로는 우울과 불안이 서로 다른 특성의 심리적 요인이라 는 주장을 한 연구자(S.-M. Kwon, 1996; J. Lee \& Chung, 2009) 도 있어, 추후 연구에서는 두 심리적 변인을 구분하여 살펴봄 으로써 청소년의 내재화 문제의 영향을 다각도에서 접근하는 것이 필요하다. 둘째, 분석대상을 고등학생으로 한정하여 연 구의 결과를 전체 청소년으로 일반화하는데 한계가 있다. 뿐 만 아니라 가정 내 경제적 박탈은 고등학교 시기 이전부터 경 험할 수 있는 환경적 요인이기 때문에, 박탈경험의 지속기간 에 따라 청소년시기에 미칠 수 있는 누적 효과를 고려할 필요 가 있다. 추후 연구에서는 경제적으로 박탈된 경험의 장기적 인 영향력을 파악하기 위해서는 분석 대상으로 아동기까지 확 대한 종단연구를 실시할 필요가 있다. 셋째, 본 연구에서는 청 소년의 행복감에 영향을 미치는 예측변수로 위험요인에 보다 초점을 두어 살펴보았다. 그러나 선행연구에서 행복감에 긍 정적 영향을 미치는 변인으로 밝혀진 부모의 긍정적인 양육 태도, 자기효능감, 또래관계의 질, 자아탄력성 등(Y. H. Kim, 2010; S.-W. Kwon, Lee, \& Song, 2012; Raboteg-Saric \& Sakic, 2014)은 위험요인이 행복감에 미치는 영향력을 완충시킬 것 으로 생각해볼 수 있다. 따라서 추후 연구에서는 위험요인 뿐 아니라 보호요인을 함께 고려하여 연구가 이루어진다면 보다 풍부한 시사점을 제시할 수 있을 것으로 생각된다.

이러한 제한점에도 불구하고, 본 연구의 결과는 청소년 행 복감 증진을 위해 경제적 요인을 살펴보았을 뿐 아니라, 경제 적 요인으로 소득수준을 기반으로 한 빈곤개념보다 다차원적 인 영역을 반영한 박탈경험을 고려하여 청소년 행복감과의 관 계를 살펴봄으로써, 청소년 행복감에 대한 이해를 높이는데 
기여하였다. 또한, 청소년의 행복한 삶을 위해서 실질적인 복 지정책을 수립하고 지원하는 것이 필요하며, 가족 갈등을 예 방하고 가족기능을 강화할 수 있는 가족 단위의 상담 및 중재 프로그램 개발 및 지원과 청소년 개인의 심리적 적응을 돕기 위한 상담 프로그램 개발 및 지원이 필요하다는 점을 밝혔으 며, 이에 필요한 기초자료를 제공하였다는 점에서 본 연구의 의의가 있다.

\section{Notes}

This article was presented at the 2018 Annual Spring Conference of the Korean Association of Child Studies.

\section{Conflict of Interest}

No potential conflict of interest relevant to this article was reported.

\section{References}

\section{In English}

Achenbach, T. M. (1991). Manual for the Child Behavior Checklist/4-18 and 1991 profile. Burlington, MD: University of Vermont.

Axelson, D. A., \& Birmaher, B. (2001). Relation between anxiety and depressive disorders in childhood and adolescence. Depression and Anxiety, 14(2), 67-78. doi:10.1002/da.1048

Brooks-Gunn, J., \& Duncan, G. J. (1997). The effects of poverty on children. The Future of Children, 7(2), 55-71.

Brown, S. L. (2004). Family structure and child well-being: The significance of parental cohabitation. Journal of Marriage and Family, 66(2), 351-367. doi:10.1111/j.1741-3737.2004.00025.x

Buist, K. L., Verhoeven, M., Hoksbergen, R., Ter Laak, J., Watve, S., \& Paranjpe, A. (2017). Associations of perceived sibling and parent-child relationship quality with internalizing and externalizing problems: Comparing Indian and Dutch early adolescents. The Journal of Early Adolescence, 37(8), 11631190. doi:10.1177/0272431616653473

Chaplin, T. M. (2006). Anger, happiness, and sadness: Associations with depressive symptoms in late adolescence. Journal of Youth and Adolescence, 35(6), 977-986. doi:10.1007/ s10964-006-9033-x
Cheng, H., \& Furnham, A. (2003). Personality, peer relations, and self-confidence as predictors of happiness and loneliness. Journal of Adolescence, 25(3), 327-339. doi:10.1006/ jado.2002.0475

Conger, R. D., \& Elder, G. H. (1994). Families in troubled times: Adapting to change in rural America. Retrieved from ERIC database. (ED391634)

Conger, R. D., Ge, X., Elder, G. H., Lorenz, F. O., \& Simons, R. L. (1994). Economic stress, coercive family process, and developmental problems of adolescents. Child Development, 65(2), 541-561. doi:10.1111/j.1467-8624.1994.tb00768.x

Cummings, C. M., Caporino, N. E., \& Kendall, P. C. (2014). Comorbidity of anxiety and depression in children and adolescents: 20 years after. Psychological Bulletin, 140(3), 816-845. doi:10.1037/a0034733

Derdikman-Eiron, R. U. T. H., Indredavik, M. S., Bratberg, G. H., Taraldsen, G., Bakken, I. J., \& Colton, M. (2011). Gender differences in subjective well being, self-esteem and psychosocial functioning in adolescents with symptoms of anxiety and depression: Findings from the Nord-Trøndelag health study. Scandinavian Journal of Psychology, 52(3), 261-267. doi:10.1111/j.1467-9450.2010.00859.x

Diener, E. (2000). Subjective well-being: The science of happiness and a proposal for a national index. American Psychologist, 55(1), 34-43. doi:10.1037/0003-066x.55.1.34

Gariepy, G., Elgar, F. J., Sentenac, M., \& Barrington-Leigh, C. (2017). Early-life family income and subjective well-being in adolescents. PloS one, 12(7), e0179380. doi:10.1371/ journal.pone. 0179380

Hanson, M. D., \& Chen, E. (2007). Socioeconomic status and health behaviors in adolescence: A review of the literature. Journal of Behavioral Medicine, 30(3), 263-285. doi:10.1007/ s10865-007-9098-3

Holder, M. D., \& Coleman, B. (2008). The contribution of temperament, popularity, and physical appearance to children's happiness. Journal of Happiness Studies, 9(2), 279302. doi:10.1007/s10902-007-9052-7

Holder, M. D., \& Klassen, A. (2010). Temperament and happiness in children. Journal of Happiness Studies, 11(4), 419-439. doi:10.1007/s10902-009-9149-2

Joronen, K., \& Åstedt-Kurki, P. (2005). Familial contribution to adolescent subjective well-being. International Journal of Nursing Practice, 11(3), 125-133. doi:10.1111/j.1440-172x. 2005.00509.x

Kline, R. B. (2015). Principles and practice of structural equation modeling. New York: Guilford publications.

King, N. J., Ollendick, T. H., \& Gullone, E. (1991). Negative affectivity in children and adolescents: Relations between anxiety and depression. Clinical Psychology Review, 11(4), 441-459. doi:10.1016/0272-7358(91)90117-d 
Letourneau, N. L., Duffett-Leger, L., Levac, L., Watson, B., \& YoungMorris, C. (2013). Socioeconomic status and child development: A meta-analysis. Journal of Emotional and Behavioral Disorders, 21(3), 211-224. doi:10.1177/1063426611421007

McAuley, C., \& Layte, R. (2012). Exploring the relative influence of family stressors and socio-economic context on children's happiness and well-being. Child Indicators Research, 5(3), 523-545. doi:10.1007/s12187-012-9153-7

Neumann, A., Van Lier, P. A. C., Frijns, T., Meeus, W., \& Koot, H. M. (2011). Emotional dynamics in the development of early adolescent psychopathology: A one-year longitudinal study. Journal of Abnormal Child Psychology, 39(5), 657669. doi: doi:10.1007/s10802-011-9509-3

Raboteg-Saric, Z., \& Sakic, M. (2014). Relations of parenting styles and friendship quality to self-esteem, life satisfaction and happiness in adolescents. Applied Research in Quality of Life, 9(3), 749-765. doi:10.1007/s11482-013-9268-0

Sheeber, L., Hops, H., Alpert, A., Davis, B., \& Andrews, J. (1997). Family support and conflict: Prospective relations to adolescent depression. Journal of Abnormal Child Psychology, 25(4), 333-344. doi:10.1023/A:1025768504415

Uusitalo-Malmivaara, L., \& Lehto, J. E. (2013). Social factors explaining children's subjective happiness and depressive symptoms. Social Indicators Research, 111(2), 603-615. doi: 10.1007/s11205-012-0022-z

Wadsworth, M. E., \& Compas, B. E. (2002). Coping with family conflict and economic strain: The adolescent perspective. Journal of Research on Adolescence, 12(2), 243-274. doi:10.1111/1532-7795.00033

Walper, S. (2009). Links of perceived economic deprivation to adolescents' well-being six years later. Journal of Family Research, 21(2), 107-127.

Wilkinson, R. B., \& Walford, W. A. (1998). The measurement of adolescent psychological health: One or two dimensions?. Journal of Youth and Adolescence, 27(4), 443-455. doi:10.1023/A:1022848001938

Zheng, X. (2016). A study on relationship between depression and subjective well-being in college student. Psychology, 7, 885888. doi:10.4236/psych.2016.76090

\section{In Korean}

Ahn, D. (2012). A study on happiness of adolescents in Korea and China. Korean Journal of Educational Research, 50(1), 227255.

Chung, H.-J., \& Choi, I.-S. (2004). Relationships among family conflicts, psychological adjustment, and career attitude maturity perceived by adolescent. The Korea Journal of Counseling, 5(3), 773-791.

Institute for Social Development Studies, Yonsei University. (2010).
Hangug eolini-cheongsonyeon haengbogjisuui guchuggwa gugjebigyoyeongujosa gyeolgwabogoseo [한국 어린이-청소년 행복지수의 구축과 국제비교연구조사 결과보고서]. Seoul: Institute for Social Development Studies, Yonsei University.

Joung, E. H., Choi, S.. Lee, S.-K., \& Ha, T.-J (2013). Aspects of child poverty (Report No. 2013-30). Seoul: Korea Institute for Health and Social Affairs.

Jung, E, S, \& Cho, H. I. (2009). The effects of parental care and overprotection on subjective well-being and depression: The role of hardiness as mediators. The Korean Journal of Counseling and Psychotherapy, 21(1), 209-227.

Kang, H. (2013). The effect of cumulative risk of adolescents on their depression and anxiety. The Korean Journal of Youth Studies, 20(9), 175-197.

Kang, H. (2015). The impact of a parental support and a selfactualization need on an academic satisfaction and a psychological euphoria. Forum for Youth Culture, 43, 7-30.

Kim, E. (2015). Measuring multidimensional child poverty in Korea: New approach to measure material deprivation of children. Korea Social Policy Review, 22(3), 105-137 .doi:10.17000/kspr.22.3.201509.105

Kim, H.-J. (2017). The structural analysis on related variables to youth psychological well-being. The Journal of Play Therapy. 21(3), 175-190.

Kim, J.-I. (2009). The impact of economic pressure on school life and problem behavior of poor children: Focusing on family risk variables and verification of mediating effect of negative parental role. Journal of Korean Society of Child Welfare, 28, 7-43.

Kim, J., You, J.-W., \& Song, I. H. (2015). Effect of socioeconomic deprivation on depressive mood: Analysis of the moderating effect of age. Health and Social Welfare Review, 35(3), 4270. doi: 10.15709/hswr.2015.35.3.42

Kim, K.-J. \& Kim, H.-J. (2014). The study on the current status of Korean childrne's and young people's rights IV (Report No. 14-R14-2). Sejong: National Youth Policy Institute.

Kim, S. S. (2016). A study on the factors affecting the children's happiness and their depression. Journal of School Social Work, 34, 91-108. doi:10.20993/jssw.34.5

Kim, U., Park, Y.-S., \& Baak, S.-Y. (2012). Factors the influence happiness and unhappiness among adolescent and their parent: Indigenous psychological analysis. The Korean Journal of the Human Development, 19(2), 197-225.

Kim, Y. H. (2010). The relationship of daily stress and egoresilience to the happiness of children and adolescent. Korean Journal of Youth Studies, 17(12), 287-307.

Kim, Y.-H., \& An, S. (2008). Family cohesion, family adaptability, parent-adolescent communication, family conflict, and adolescents' depression \& delinquency. Korean Journal of Youth Studies, 15(2), 1-30. 
Korea Institute of Social and Health Affairs., \& Seoul National University Institute of Social Welfare. (2012). Korea Welfare Panel Study $7^{\text {th }}$ survey [Data file and code book]. Retrieved from https://www.koweps.re.kr:442/data/data/list.do

Kwon, S.-M. (1996). Relationship between depression and anxiety: Their commonness and difference in related life events and cognitions. Psychological Science, 5(1), 13-38.

Kwon, S.-W., Lee, A.-H., \& Song, I,-H. (2012). A study on adolescent happiness: Application of the adolescent resilience model. Studies on Korean Youth, 23(2), 39-72.

Lee, H. (2016). Determinants of subjective well-being among Korean youth: Focusing on socio-demographic and social capital factors, Studies on Life and Culture, 39, 151-186.

Lee, J. \& Chung, H. (2009). Cognitive triad: Relationship with depression and anxiety in children. Cognitive Behavior Therapy in Korea, 9(2), 49-63.

Lee, J.-M., \& Lee, Y.-H. (2007). The effects of perceived parental conflict, maternal behavior, and social support on children's subjective well-being: A structural equation modeling analysis. The Korean Journal of Developmental Psychology, 20(4), 33-58.

Lim, H.-J., Baek, H.-J., Kim, H.-C., \& Kang, H.-C. (2012). 2012 International survey on youth's values (Report No. 2012-22). Seoul: Ministry of Gender Equality \& Family.

Lim S. H., \& Kim S. (2016). The effects of housing poverty on adolescents' subjective well-being. Journal of the Korean Society of Child Welfare, 56, 133-164.

Noh, C.-R., \& Kim, S.-H. (2012). The relationship between studyrelated stress, perceived school grades, and psychological well-being among middle school students. -Mediating effects of personal factors and social support on self-esteem, depression, and anxiety. Journal of the Korean Society of Child Welfare, 39. 39-68.

Noh, P., \& Yoon, H. (2007). The effect of the economic strain, family function \& social support on middle school students' problem behaviors. Journal of School Social Work, 12, 23-46.

Oh, K. J., Lee, H., Hong, K.-E., \& Ha, E. H. (1998). Korean Child Behavior Checklist(K-CBCL). Seoul: ChungAng Aptitude Publishing Co.

Park, E., \& Baek, S. (2017). The mediating effects of optimism on the relationship between parenting behavior and happiness perceived by adolescent. Korean Journal of Counseling, 18(4), 149-166.

Park, S. (2013). The influence of physical resource loss and gain on well-being of people in low-income and the mediating role of economic stability. Korean Journal of Social Welfare, 65(2), 335-356.

Park, S.-Y., \& Lee, H. (2013). Determinants of subjective wellbeing among Korean adolescents. Stress, 21(2), 73-84.
Shim, W.-Y. (2014). Mediation effects of self-compassion in the relationship between social anxiety and happiness of elementary school students. The Journal of Elementary Education, 27(1), 83-102.

Sihn, M., Jeon, S. H., \& Yoo, M. S. (2010). A study on factors affecting life satisfaction and wellbeing in youth. Korean Journal of Youth Studies, 17(9), 131-150.

Shin, M., Chung, K.-M., \& Kim, E.-S. (2012). Gender differences in depression and anxiety among Korean adolescents: Onset and developmental change. The Korean Journal of Clinical Psychology, 31(1), 93-114. doi:10.15842/ kjcp.2012.31.1.005

Suh, J. W., \& Jung, Y. T. (2014). Comparison of influences of variables on subjective happiness in the youth: Personal capability, family environment and school environment. Korean Journal of Youth Studies, 21(8), 265-292.

Sung, E., \& Kim, G. (2013). The structural relationships analysis among happiness, personal traits, and environmental traits of adolescents using the structural equation modeling. Studies on Korean Youth, 24(4), 177-202.

Yoo, I.-Y., \& Lee, J.-A. (2013). Subjective health status and happiness of adolescents in multi-cultural family environments. Journal of the Korean Society of Living Environmental System, 20(5), 699-707.

Yoon, H.-M. (2005). Effects of economic strain and family conflict on children's adjustment: Focused on interaction effects of children's stress and coping strategies. Korean Journal of Social Welfare, 57(3), 133-164.

Yoon, G. J. (2012). The effects of goals, satisfaction, self-efficacy and depression on the happiness of early childhood pre-service teachers. Journal of Korean Child Care and Education, 8(3), 113-131.

Youm, Y. S., \& Kim, K. M. (2017) Hangug eolini.cheongsonyeon haengbogjisu[한국 어린이·청소년 행복지수](Report No. 2017-9). Seoul: Korean Bang Jeong-hwan Foundation.

\section{ORCID}

Sae-Young Han http://orcid.org/0000-0001-8207-5927

Ah-Reum Han https://orcid.org/0000-0003-4715-6494

Received April 30, 2018 Revision received June 24, 2018

Accepted August 2, 2018 